\title{
Naissance des Antigones
}

À la question «Où lire la genèse des poèmes d'Henry Bauchau ? ", ce colloque entend fournir des réponses articulées et approfondies. Certes, il faut des années pour qu'un collectif de recherche bien équipé et bien entraîné vienne à bout des gribouillis et des fouillis de feuillets dans lesquels un écrivain laisse trace du ferment intérieur de son inspiration (voir Proust, Flaubert ou Zola). Mais il est certain qu'interroger conjointement les journaux ${ }^{1}$, la bibliothèque et les manuscrits sont les fondements obligés, incontournables, pour une histoire génétique de la poésie d'Henry Bauchau.

Ma modeste contribution à cette entreprise se limite à l'étude de quelques feuillets. Ni journaux, ni bibliothèque donc, mais un gros plan sur un simple geste d'écriture, ou mieux, un geste en suspens, bégayant, hésitant, revenant sur ses pas, inlassablement. Les quelques manuscrits observés ici touchent au seul poème «Les deux Antigone». Selon le témoignage du journal Les Années difficiles (AD, p. 411), ils ont été travaillés entre le 25 août et le 20 octobre 1982 :

L'écriture d'un de ces poèmes : «Les deux Antigone » m'a pris deux mois et m'a mené à la limite de mes forces. Ce poème, qui demeure à mes yeux le plus attentif de ceux que j'ai écrits, a eu une grande importance dans l'évolution de mon œuvre en ramenant dans mes perspectives d'écriture deux personnages auxquels je m'intéressais depuis longtemps : CEdipe et Antigone. (EE, p. 92.)

\footnotetext{
1 Déjà partiellement sondés par Geneviève Henrot, "La Naissance du poème ", dans Henry Bauchau poète. Le Vertige du seuil, Genève, Droz, 2003, pp. 19-41. Voir aussi Deborah Gabriel, «Les journaux, l'autre voix de l'écriture », dans Jacques Poirier et Myriam Watthee-Delmotte (dir.), Pierre Jean Jouve et Henry Bauchau: les voix de l'altérité, Dijon, Éditions universitaires de Dijon, 2006, pp. 199-212; Catherine Mayaux, «La dictature du poème, ou le poème cet inconnu. L'expérience poétique chez Henry Bauchau d'après ses journaux ", Nu(e), n³5, 2007, pp. 201-211 ; Geneviève Henrot, «La voix de l'ombre. Pouvoirs du rêve dans les journaux d'Henry Bauchau », ibid., pp. 249-260 ; Annie Pibarot, "La présence du rêve dans les journaux d'Henry Bauchau », Revue Internationale Henry Bauchau, n5, 2013, pp. 141-150.
} 
Le nom d'Antigone apparait également dans d'autres poèmes: "Regards sur Antigone», "Le monologue d'Antigone», "Liberté d'Antigone », "Le Chœur», "Antigone à la lance » sont compris dans le recueil Nous ne sommes pas séparés (les épreuves des poèmes pour Actes Sud, 28 mars 2006 font l'objet des Dossiers n 4 et 6 du Fonds Henry Bauchau de l'Université catholique de Louvain). Ou encore, dans Exercice du matin (Dossier $\mathrm{n}^{\circ} 4$ ), "Stèle pour Antigone » (A1292) hésite un peu, là aussi, sur le titre : "L'épiphanie d'août» (A1295-A1299), "L'inattendu d'août» (A1300-A1329). Il existe aussi un «Passage d'Antigone» (A1988-A1991).

Mais, sans prétendre aujourd'hui suivre du doigt une histoire d'Antigone dans l'œuvre poétique ${ }^{2}$, qui fasse pendant à l'Antigone tant étudiée dans le roman homonyme ${ }^{3}$, je voudrais pour cette fois me concentrer sur un seul poème. La génétique textuelle, encore plus que la stylistique ou la linguistique, entraîne le chercheur à une attention à la fois microscopique et radiographique des textes, qui impose de circonscrire un champ opératoire très restreint.

Les cotes de catalogation des feuillets sélectionnés tiennent vraisemblablement compte de l'ordre dans lequel ils se présentaient dans leur chemise ou carton à l'arrivée au Fonds Henry Bauchau. Les manuscrits proprement dits se succèdent dans une séquence ordonnée relativement cohérente, compatible avec les réécritures partielles que comporte chaque feuillet. Sauf à voir apparaître en premier un tapuscrit (A700) correspondant au texte publié en 1986. C'est un peu comme si, dans le dossier homogène et continu constitué par ces feuillets, la version

\footnotetext{
${ }^{2}$ On en trouvera un début de parcours dans Geneviève Henrot, « Nébuleuse Antigone. Formation mosaïque d'une figure mythique dans la poésie d'Henry Bauchau », dans Massimo Peri (dir.), Autografi letterari romanzi e neogreci. Problemi teorici, editoriali e interpretativi. Due giornate di studi in memoria di Filippo Maria Pontani, Università degli Studi di Padova, 24-25 ottobre 2013, à paraître.

3 Pensons, entre autres, à Annie Pibarot, «Le motif de la mendiante ou "le lieu de l'écrit" ", dans Marc Quaghebeur (dir.), Les Constellations impérieuses d'Henry Bauchau. Colloque de Cerisy 21-31 juillet 2001, Bruxelles, AML Éditions/ Labor, "Archives du futur », 2003, pp. 273-287 ; Anne Davenport, "Antigone dans le contexte américain », Ibid., pp. 365-383; Christiane Chaulet-Achour, "Le personnage d'Antigone: une approche poétique de l'androgynie », dans Catherine Mayaux et Myriam WattheeDelmotte (dir.), Henry Bauchau, écrire pour habiter le monde, Saint-Denis, Presses Universitaires de Vincennes, "L'Imaginaire du texte», 2009, pp. 227-238; Emilia Surmonte, Antigone, La sphinx d'Henry Bauchau. Les enjeux d'une création, Peter Lang, 2011.
} 
finale tapuscrite devait servir de "couverture thématique », de "belle page » au carton de brouillons ${ }^{4}$.

N'ayant pu suffisamment approfondir l'état matériel des manuscrits, je ne me chargerai certes pas ici de les établir (au sens technique du terme), mais seulement de les exploiter directement à des fins poétiques. Partant, la seule orientation téléologique envisagée tiendra à l'état final du poème tel qu'il a été publié en 1986 et légèrement modifié par la suite.

Les questions poétiques, posées au niveau phrastique, voire intraphrastique (de l'ordre du syntagme), viseront à enquêter sur le personnel du poème (je, tu, elle), et plus spécifiquement sur le statut actanciel et spirituel d'Antigone dans l'organigramme du poème. Il me semble en effet fructueux de discuter, pour le moins, les deux points suivants :

1) la question des identités, et partant, des identifications qui s'établissent chemin faisant entre les séries de personnages ;

2) la question des qualités qui circulent dans un jeu d'échanges réciproques. C'est à ce jeu d'équivalence et de prédication que le nom propre du thème-titre ("Antigone») gagne sa vocation à supporter le pluriel.

\section{Analyse du corpus}

\section{Analyse matérielle du dossier génétique des «Deux Antigone 》}

À s'en tenir au relevé du Fonds Henry Bauchau de l'Université catholique de Louvain-La-Neuve, voici les feuillets répertoriés comme constitutifs du Dossier n ${ }^{\circ}$, concernant «Les deux Antigone» (A699A721) : les feuillets A702 à A712 présentent la version manuscrite «en travail », les feuillets A713-723 montrent plusieurs versions tapuscrites datées du 25 août-20 octobre 1982. S'y ajoutent, toujours dans le Dossier $\mathrm{n}^{\circ} 3$, quelques dernières retouches au tapuscrit définitif envoyé de Montour à Actes Sud, fin août 1986 (A825-826, 1982); ainsi qu'une copie de la version publiée par Actes Sud en 1986, dédicacée à

\footnotetext{
4 Cette version placée en évidence intègre entre autres la correction d'«âge » en «temps » dont on peut voir la trace manuscrite sur le feuillet A713, cependant que les copies A715-716 et A717-718 ne portent pas de correction, la première étant par ailleurs vraisemblablement la copie carbone de la seconde.
} 
Dominique Stein (A721-A722 et son carbone A881-A 882, 1982). Voilà donc, en synthèse, le corpus de travail. Il comprend essentiellement des types de documents que les généticiens classifient en «états rédactionnels » (les manuscrits du travail de rédaction proprement dite), en «états pré-éditoriaux» (les dactylographies de mise au net préparatoires à l'édition) et en "états éditoriaux » (les jeux d'épreuves et leurs corrections). Auxquels s'ajoutent, bien sûr, les éditions effectives, qui peuvent subir, elles aussi, des remaniements dans la contextualisation séquentielle des poèmes les uns par rapport aux autres ${ }^{5}$. Quant à d'éventuels « états pré-rédactionnels » du poème (notes, esquisses, listes, carnets, idée initiale), ils sont à chercher, faute de traces manuscrites connues à ce jour, dans le journal des Années difficiles.

Est donnée pour acquise la transcription diplomatique ${ }^{6}$, présentée en parallèle, des folios A702 à A712. Au vu du dossier des avant-textes ainsi construits, le champ des rédactions se circonscrit pour l'essentiel sur quelque dix pages manuscrites, à savoir principalement sept versions manuscrites « en travail». Si cette chronologie devait subir des révisions à la suite d'une analyse matérielle plus approfondie, je gage qu'une quelconque variation de la chronologie n'en porterait pas pour autant atteinte à la configuration des rôles actantiels ${ }^{7}$ tels qu'ils se font jour sur

\footnotetext{
${ }^{5}$ Publié pour la première fois dans Poésie 1950-1986 (Arles, Actes Sud, 1986, p. 237 et ss.), le recueil Les deux Antigone, constitué de 17 poèmes, et dédié à Laure, réapparaît dans Heureux les Déliants. Poèmes 1950-1995 (Lecture de Geneviève Henrot, Bruxelles, Labor, 1995, pp. 49-50); en 2006, le poème Les deux Antigone change de dédicace (à Jean-Pierre) et est incorporé dans le recueil Nous ne sommes pas séparés. Poésie, Arles, Actes Sud, 2006, pp. 44-45. En 2009, quatorze des dix-sept poèmes sont repris dans Poésie complète, dont Les deux Antigone, pp. 255-256.

$6 \mathrm{La}$ présentation diplomatique (ou ultra-diplomatique) se donne pour tâche de reproduire la disposition spatiale de l'écriture de manière aussi mimétique que possible. Les règles typographiques adoptées ici sont les suivantes: l'alignement vertical des réécritures supra ou sub-linéaires rend compte de la gestion originale de l'espace paginal ; le barré simple indique la biffure; les soufflets $<>$ signalent des ajouts. Les crochets [] encadrent une hypothèse de déchiffrement.

${ }^{7}$ Voir Jean Dubois et al., Dictionnaire de linguistique, Paris, Larousse, 2002, p. 14, pour un panorama des acceptions du terme «actant»: «1) Le nom d'actant est donné à celui qui fait l'action indiquée par le verbe (intransitif) ou le groupe verbal formé du verbe et de son objet (transitif) : il répond à la question implicite Que fait X ? X est l'actant ou l'agent de l'action. 2) Dans l'analyse structurale du récit, l'actant est le protagoniste de l'action, distinct du bénéficiaire, au bénéfice de qui se fait l'action; actant et bénéficiaire peuvent se confondre dans la même personne. 3) L. Tesnière appelle
} 
le mode équatif au niveau d'abord syntagmatique et ensuite phrastique. C'est donc un choix de méthode, inspiré par l'état (toujours) potentiellement provisoire des catalogations et des datations, qui suggère de faire état de paradigmes d'équivalence, sans aucune visée téléologique ou diégétique. Il est par ailleurs plus significatif, à mon sens, d'observer tout d'abord, d'entrée de jeu, un profil de réécriture intrapaginale, qui d'ailleurs ne semble guère se transformer au fil des feuillets.

Analyse génétique : le processus de réecriture "par vagues 》

Dans la suite des feuillets, sauf à constater le retour ferme, constant, presque inaltéré des deux premiers vers («Ainsi l'âge nous fait l'un pour l'autre Antigone...»), la mise en place de la première moitié du poème est manifestement plus laborieuse que celle de la seconde moitié, pour laquelle les manuscrits présentent bien moins de versions. Si l'on compare, feuillet après feuillet, le travail de chaque vers, dans l'ordre de son apparition, il est visible que vaincre la paralysie de la page blanche requiert les plus grandes énergies aux «accords» des premiers vers, cependant que la suite, une fois campé le cadre, n'exige plus qu'un écoulement du récit.

Le profil génétique esquissé ici réclame son individualité. Il est frappant de constater combien, au-delà de quelques grands schèmes d'écriture, tels qu'ils ont été mis au jour dans les premières années de la génétique textuelle (les écritures «à procès 》 et les écritures «à processus $»)^{8}$, chaque dossier d'auteur présente des traits particuliers qui en déterminent la physionomie singulière. Ainsi de Flaubert, de Balzac, de Proust, de Zola, ou encore de Picasso ou d'Andrée Chédid'. Pour avoir contemplé quelques copies au net aimablement envoyées par Henry Bauchau il y a quelques années, je craignais sincèrement qu'il n'y aurait pas grand-chose à dire sur ces pages proprettes, gentiment soignées comme un cahier d'écolier appliqué, un peu comme celles de

actants les unités désignant les êtres ou les choses qui, d'une manière ou d'une autre, même en tant que simples figurants, participent au procès exprimé par le verbe. Les verbes sont ainsi caractérisés par le nombre d'actants qu'ils peuvent avoir.

8 Almuth Grésillon, Éléments de critique génétique, Paris, P.U.F., 1994 ; Michel Contat et Daniel Ferrer (dir.), Pourquoi la critique génétique? Méthodes, théories, Paris, CNRS, 1998.

${ }^{9}$ Louis Hay (dir.), Les Manuscrits des écrivains, Paris, Hachette/CNRS Éditions, 1993 ; Marie-Odile Germain et Danièle Thibault (dir.), Brouillons d'écrivains, Paris, Bibliothèque Nationale de France, 2001. 
Jean-Paul Sartre ou de Jean-Pol Goux. Aussi le dossier des «Deux Antigone » a-t-il constitué pour moi une surprise légère, mais réjouie: dans son apparente "propreté », l'ensemble des brouillons n'en présentait pas moins des traces éloquentes d'un type spécifique d'écriture en travail!

Seule une description textuelle est actuellement possible, tant qu'il manque une histoire de la graphie, une histoire des fournitures en papier, une histoire des instruments (stylos à bille, stylos à encre, crayons), et aussi une description des rituels d'écriture. Pour autant, on peut constater une «foulée » d'écriture limitée à deux, trois, quatre vers, que le poète reprend et modifie dans le champ de bataille de la même page. Là où un Flaubert travaille par feuillet entier, qu'il recopie complètement à chaque étape et puis barre d'une croix de saint André pour signaler sa réutilisation, là où un Proust déverse d'infinies annexes en marge, en page de gauche puis sur paperoles pliées en accordéon, Bauchau, laborieux, parcimonieux, médite lentement chaque vers, cale, rebrousse chemin, tente des reformulations et tâte surtout les déplacements syntactiques et les équivalences syntagmatiques. Il faudra voir maintenant pourquoi et comment ce style de travail essentiellement paradigmatique s'avère révélateur d'un ordonnancement viscéral du personnel de la fiction; celui-ci fonctionne par types et occurrences, par incarnations et figurations, par identités singulières et qualités en partage. L'analyse qui suit s'impose une lecture prioritairement immanente et systémique, ignorant volontairement, par méthode, les autocommentaires d'Henry Bauchau dans son journal.

Analyse poétique

À voir où le texte «cale » dans ses réécritures, où est rayé le sillon du disque, c'est de toute évidence la part mythique du poème qui donne du fil à retordre, là où se rencontrent, se télescopent et tentent de se fondre :

- le mythe culturel d'Antigone, actualisé dès le titre et la rime du premier vers ;

- le mythe personnel de la petite enfance, avec ses figurations tutélaires, réelles ou fictives, de Mère et de Mérence ;

- le mythe de la Sibylle qui, comme on sait, jette un pont entre l'Antiquité et le vécu personnel, autobiographique. 
C'est la motivation actancielle de cette fusion et ses creusets syntagmatiques qui nous retiendront surtout ici.

Dans la première moitié du poème, Henry Bauchau travaille, disionsnous, sur de petits groupes de vers (deux - trois - quatre) correspondant à un embryon de phrase; il les reprend inlassablement, à plusieurs reprises sur la même page ${ }^{10}$ :

L'age

Ainsi l'age ns fait l'1 pour l'autre Antigone $<$ quête $>$

Non point l'age mais l'ame en rêve du royaume

SurEt des genoux sacrés de mère en beauté jeune $<$ au seuil de joie $>\quad<$ deuil $>$

La fut l'errance et fut Merence avant le jeune

De la statue de joie ancienne toute en psaume

Statue de Mèrence...

la fut le seuil avant le deuil

de la stature de Mèrence sur le seuil

Statue de psaume et de joie

Psaume de joie ancienne édit de deuil

O statures Mèrence haute sur le Seuil

$<$ ordonnance de deuil $>$

Statue de joie ancienne en larme statue de

psaume

Et de comptine dont il faudra qu'on ressuscite

Dont il faudra qu'un jour en plein jour on existe tout en cloitre de larmes dence en genoux d'Antigone

Dont il faudra qu'un jour prochain on ressuscite (A702)

Du point de vue simplement graphique, l'extrême rareté des ratures (sur ce feuillet, deux seulement, sur au v.3 et douce-plus bas) laisse supposer des repentirs très faibles, hésitants, peu convaincus, qui gardent en réserve la première formule venue sous la plume. La rature semble

10 La transcription proposée se tient à la graphie du poète, celle-ci dût-elle être incomplète ou erronée : il est du reste particulièrement significatif d'observer que l'accent sur le premier « $\mathrm{e}$ » de « Mérence » apparaît régulièrement grave plutôt qu'aigu, comme celui de « mère ». 
signaler une impulsion immédiate à reprendre da capo, dans le droit fil de la ligne (comme plus loin). En revanche, lorsque le vers venu en entier est aussitôt repensé, l'inscription sub-linéaire peut encore accueillir la nouvelle formulation (totale ou partielle), sans devoir recourir à la rature, mais optant plutôt pour une mise en paradigme vertical, comme dans :

Psaume de joie ancienne édit de deuil

$$
\text { ordonnance de deuil }
$$

Par contre, l'inscription supra-linéaire fait imaginer une correction advenant quand le poète revient après coup sur le groupe de vers déjà écrit(s), l'espace sub-linéaire se trouvant donc déjà occupé par du texte : c'est le cas de <deuil > à la place de <jeune $>$, ou de <quête $>$ venant remplacer $<$ rêve $>$. Ce dernier exemple montre combien la correction peut n'avoir rien de définitif, à suivre du doigt son périple : rêve (A701) $>$ quête $($ A702) $>$ vœu secret $($ A703) $>$ désir debout (A704), quête $<$ rêve> (A705), pour enfin établir « quête » dans la version finale.

Une fois qu'on a pris acte du processus de mise en paradigme résultant des diverses réécritures, apparaissent au gré des reprises de nombreux éléments de chronotopes bauchaliens qui nous sont familiers :

- le vecteur du temps (chronos) affronte sur un perron ${ }^{11}$ un avant et un après, le premier enfantin, insouciant et rieur, l'autre muet de larme et de deuil ; entre l'un et l'autre, une scène pétrifiée, figée dans sa dureté de pierre, perron, socle et statue ;

- le vecteur du logos discerne lui aussi un avant tout en parole heureuse et insouciante («comptine») et sereinement festive (les "psaumes» des dimanches), dans la quiétude de la «langue originelle »; alors que l'après sombre dans le mutisme et le silence, la fermeture intérieure, bref dans une langue "sibylle»; de l'un à l'autre, l'agent paralysant d'un « édit»/« ordonnance», à savoir une parole ultime, capitale, comme peut l'être une peine infligée pour une faute grave ;

- le vecteur de l'espace (cosmos) ouvre à partir de ce même seuil un paysage fixe (pétrifié) tout en verticale, bien différent de l'horizon d'errance où CEdipe et Antigone circulent en rond. En haut de l'escalier,

\footnotetext{
${ }^{11}$ On sait quelle importance thématique et symbolique le seuil acquiert dans l'imaginaire bauchalien : voir Geneviève Henrot, Henry Bauchau poète. Le Vertige du Seuil, Genève, Droz, 2003.
} 
sur ce même seuil, immense, la figure tutélaire de l'enfance, et en bas, un demain d'expiation et de rachat, où «il faudra descendre à reculons ». À mi-mots se recompose sous nos yeux, après vingt ans, le scénario narré dans «L'Escalier bleu » et dans La Déchirure: «L'amour du monde avant sa chute dans le froid »;

- le vecteur de l'anthropos: rien d'étonnant que le poème, ce faisant, rappelle des coulisses toute cette Cour des Miracles où princes et mendiants sont confondus dans une même misère des seuils interdits (la maison d'Archennes, la ville de Thèbes) : les princes, Olivier et Paul, mais aussi l'Homme noir, avant CEdipe et Antigone; et les mendiants, ces pauvres du canton, avant nos exilés. La réactivation de la scène d'enfance à l'occasion d'un rêve mythique installe le moi au carrefour de deux dimensions, ontogénétique et phylogénétique: l'enfance du je (Mère, Mérence) et celle du mythe (CEdipe et Antigone). Partant, l'ambivalence des scénarios en surimpression engage à la fois :

- un système d'équivalences féminines (Mère, Mérence, Antigone) et masculines (je, Roi Lear, (Edipe) ;

- un système d'équivalences des genres (masculin/féminin).

Comment ces systèmes affleurent-ils de l'état d'inachèvement provisoire des pages de brouillons? D'une réécriture l'autre, se font jour principalement trois forces: l'écho phonique, le gouvernement syntagmatique et la fluctuation référentielle.

Sur base de ressemblance formelle, phonique, des attractions sonores entre mots (surtout des paronomases) supportées par des parallélismes syntaxiques invitent à postuler l'équivalence sémantique des syntagmes mis en parallèle. Faisant système ou procédé avec d'autres attractions paronomasiques (comme «âge » / «âme», «quête» / 《rêve », « errance » / « Mérence », « seuil»/ « deuil», « ressuscite » / « existe»), l'attraction "stature» / 《statue » constitue le double nœud à partir duquel se lacent deux isotopies : celle du corps singulier («stature») et celle de la figuration symbolique archétypale (« statue »). 
Sur base de construction syntagmatique, des jeux d'assimilation entre blocs syntagmatiques [détermination + tête + expansion] postulent à leur tour une mise en paradigme ${ }^{12}$ de figures actancielles (ou rôles profonds).

Tantôt, le mouvement des réécritures vient placer ces syntagmes l'un sous l'autre (chose que ne donne pas à voir la version finale!), dans une équivalence paradigmatique visuelle :

\begin{tabular}{|l|l|l|}
\hline Ordre du & & \\
\hline Paradigme & $\begin{array}{c}\text { statue de Mérence }= \\
\text { [mère + errance] }\end{array}$ & $\begin{array}{c}\text { et des genoux sacrés de mère } \\
\text { en beauté jeune }\end{array}$ \\
\hline & $\begin{array}{c}\text { statue d'Antigone } \\
\text { [fille }+ \text { errance] }\end{array}$ & $\begin{array}{c}\text { toute en cloitre de larmes } \\
\text { douce en genoux d'Antigone }\end{array}$ \\
\hline
\end{tabular}

Tantôt, en vertu d'une combinaison syntagmatique, syndétique ou asyndétique, les syntagmes juxtaposés sont à considérer comme des reprises, des reformulations ou des appositions du deuxième syntagme au regard du premier. Il n'est d'ailleurs pas rare que les deux processus multiplient leur force d'attraction et conjuguent leur pouvoir d'identification : la paronomase qui relie «stature » et «statue » établit une possible compatibilité entre corporéité ("stature») et iconicité (《statue »), par quoi les deux compléments déterminatifs (" Mérence» et "Antigone ») se trouvent comme à figurer, la première une occurrence, la seconde un type, un même actant profond, l'adjuvant à la vocation maternelle.

12 Cette mise en paradigme est du reste bien plus riche que ce qui sera exploité ici, comme en témoignent les différentes occurrences dans ces brouillons : "statue de joie ancienne toute en psaume ", "Statue de Mérence », "De la statue de Mérence sur le seuil », «Statue de psaume et de joie », «Ô statures Mérence haute sur le seuil », «Statue de joie ancienne en larme », «Statue de psaume et de comptines »... 


\begin{tabular}{|c|c|c|}
\hline Ordre du & STATUE+Expansion & GENOUX+Expansion \\
\hline \multirow[t]{2}{*}{ Paradigme } & $\begin{array}{l}\text { statue de Mérence }= \\
\text { [mère }+ \text { errance }]\end{array}$ & $\begin{array}{l}\text { et des genoux sacrés de } \\
\text { mère en beauté jeune }\end{array}$ \\
\hline & $\begin{array}{l}\text { statue } d \text { Antigone }= \\
{[\text { fille }+ \text { errance }]}\end{array}$ & $\begin{array}{l}\text { toute en cloitre de larmes } \\
\text { en genoux d'Antigone }\end{array}$ \\
\hline \multirow[t]{4}{*}{ Syntagme } & $\begin{array}{l}\text { Ta stature Mérence et } \\
\text { statue d'Antigone }\end{array}$ & $\begin{array}{l}\text { D'être sous les genoux de } \\
\text { mère en beauté jeune }\end{array}$ \\
\hline & $\begin{array}{l}\text { Haute sur le seuil, } \\
\text { immense }\end{array}$ & $\begin{array}{l}\text { Serrant sa jambe immense } \underline{e t} \\
\text { statue d'Antigone }\end{array}$ \\
\hline & $\begin{array}{l}\text { mère et fille d'errance } \\
\text { et de Mérence }\end{array}$ & \\
\hline & Mérence $=$ Antigone & Mère $=$ Antigone \\
\hline
\end{tabular}

La superposition des divers syntagmes expansés opère donc entre les trois figures féminines la triangulation suivante (qui n'aura du reste rien pour étonner les lecteurs assidus d’Henry Bauchau !) :

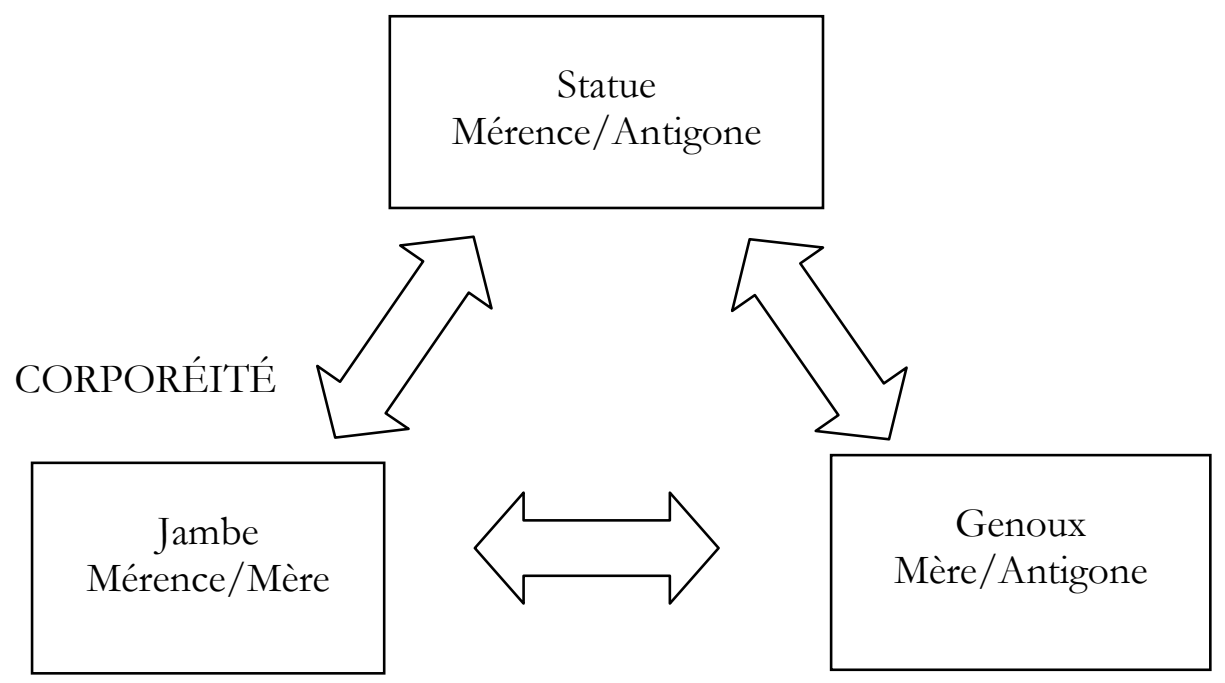


Le système d'équivalences paradigmatiques mis en place par les réécritures successives fait voyager Antigone d'une figure l'autre, puisqu'on la voit tour à tour se fondre en mère et en Mérence. Et sans doute la statue, par son pouvoir de figuration symbolique, iconique, permet-elle le transit, dans ses traits atemporels figés dans la pierre, des différents rôles féminins convoqués sur la scène du poème: statue de Mérence, statue d'Antigone, statue de mère immense.

Sa fonction tutélaire semble confiée au haut degré de sa taille, qu'implicite le substantif «stature» $(/+$ haute/ $)$, cependant que l'explicite le qualificatif «immense»; on l'infère en outre d'une perspective visuelle en contre-plongée, qui ne saisit que la partie inférieure, non seulement du corps, mais aussi de la jambe («sous les genoux »), perspective sans doute motivée elle aussi par le souvenir du point de vue enfantin; on la mesure encore à la localité de sa position (au haut de l'escalier, au seuil de la maison): on se souvient en effet de «L'Escalier bleu » («L'escalier descendait vers la ferme et les granges »).

Le corps féminin dans sa statuaire instaure, par le symbole de la sculpture, un dénominateur commun entre Mérence, mère et Antigone, à partir d'Antigone: le motif de la statue remplit donc une fonction équivalencielle. Mais on va voir qu'Antigone remplit également une fonction, non plus référentielle (désigner un personnage, fût-il mythique), mais purement qualitative.

La troisième force linguistique en jeu dans ces réécritures produit en effet une forte fluctuation de la visée référentielle, en particulier par le biais de la détermination. C'est en effet la détermination nominale, avec toute sa palette d'articles, qui permet d'«asseoir» le nom comme désignant un référent encore inconnu ( «un»), ou au contraire bien connu («le»), reporté à l'attention toute fraîche de l'interlocuteur («ce ») ${ }^{13}$, ou encore oscillant entre particulier et générique.

Nous allons voir maintenant comment la variation successive des déterminants dans un syntagme par ailleurs inchangé pousse ce même syntagme à acquérir une valeur référentielle oscillant entre le particulier

${ }^{13}$ Jacques Damourette et Édouard Pichon nomment en effet «assiette » la façon dont le déterminant précise la visée référentielle du nom qu'il contribue à déterminer: présentatoire, notoire et transitoire (Des mots à la pensée. Essai de grammaire de la langue française, Paris, D’Artrey, vol. 1, 1911-1927). 
et le générique, entre l'occurrence et le type, entre l'individu et l'archétype.

(A702) et des genoux sacrés de mère en beauté jeune.

«Des», dans cette occurrence, correspond à l'amalgame de la préposition «de» et de l'article défini simple «les»; il assure au complément déterminatif «de mère en beauté jeune» une visée référentielle particularisante, qui permettrait d'entendre "mère » presque comme un Nom propre et même de lui mettre en pensée la majuscule : «Mère ». Mais dans la version suivante, à la suite d'une rature linéaire, ce vers devient :

(A703) d'être sous dtes genoux sacrés de mère en beauté jeune

où il est clair qu'un $\mathrm{A}$ a fugitivement précédé le $-\mathrm{t}$, poussant un indéfini là où finit par prévaloir le possessif. Est-ce un lapsus calami ? «Mère» est prise entre deux apostrophes, («Antigone» au v. $1^{14}$ et «Mérence » au v. 4). Or c'est celle-ci qui, debout sur le seuil, en impose par sa stature ("haute sur le seuil»), et sa jambe immense, sous les genoux de laquelle l'enfant s'accroche (ou du moins accroche son regard). Par ailleurs, le possessif à lui seul permet d'identifier en contexte un coréférent anaphorique, qu'il s'agit de chercher ici, amont ou aval, selon l'instruction fournie par la deuxième personne du singulier, $t u$ : Antigone? Mérence? Même à oublier cette brève détermination indéfinie (ce «des» subrepticement ravalé dans la plume) qui aurait ôté toute identité singulière à «mère», pour n'en faire plus qu'un type de relation familiale, il n'en reste pas moins que ce possessif «tes » qui vient remplacer l'indéfini «des» est ambigu: il renvoie à une instance allocutaire, qui n'est pas ici la «mère », mais Mérence, et peut-être d'abord, par anaphore, Antigone ; alors que la qualification assurée par le complément épithète (qui vient après le possessif) renvoie thématiquement, lui, à mère. «Mère » en sort comme dépouillée de sa référence originelle, pour s'investir d'une valeur qualitative que rendrait tout aussi bien l'adjectif «maternels ». La mère est-elle donc moins mère que ne l'est Mérence?

\footnotetext{
${ }^{14}$ Mais sans doute doit-on aussi comprendre l'« Antigone » du premier vers comme un attribut du complément nous : «a fait de nous l'un pour l'autre une Antigone ». Bauchau pratique volontiers ce type d'ambiguïté syntaxique dans sa poésie.
} 
C'est encore une pirouette référentielle de ce genre que réussit la synecdoque du «genou» et de la «jambe» dans la version suivante, à savoir viser simultanément :

- Antigone (« tes » renvoie très vraisemblablement, par anaphore, au tu allocutaire en apostrophe dans le v.1) ;

- mère (le déterminant épithétique «en beauté jeune » est associé de façon récurrente à « mère » dans l'intratexte bauchalien) ;

- Mérence (par l'évocation de la stature immense, haute sur le seuil, d'abord attribuée à cette dernière.

(A704) [...] et le désir debout

D'être sous ces/tes/les genoux de mère en beauté jeune

Serrant sa jambe immense et statue d'Antigone

La mise en paradigme des trois déterminants confère tour à tour à l'expansion «de mère» une valence tantôt déterminative, tantôt qualificative :

- «les » : déterminative (servant à viser le référent singulier «mère », possesseur inaliénable);

- «ces » : ambigu, sa valeur présentatoire renvoie à la coprésence en contexte (dialogal) d'un «tu »; sa valeur d'exophore mémorielle pourrait en revanche viser le référent originel de mère, conforté en cela par la qualification propre qui l'accompagne («en beauté jeune») comme un attribut individuel ;

- «tes»: possessif inaliénable renvoyant à une instance «tu» qui n'est pas ici la mère, mais, en vertu des apostrophes, l'interlocutrice du poème, Antigone, ou encore Mérence.

Or, la version indéterminée finit par prévaloir, au prix d'un repentir si net qu'il recourt, exceptionnellement, à la rature de tout le vers :

(A705) Sur ses genoux sacrés de mère en beauté jeune

Sur des genoux sacrés de mère en beauté jeune

Sous la statue ancienne

Toute entière Antigone

Cette victoire disputée de l'indéfini sur le défini et le possessif fait émerger la valeur archétypale de la figure maternelle, telle qu'elle peut 
être incarnée indifféremment soit par la mère véritable, soit, mieux encore, par une mère de remplacement et de compensation comme l'imaginaire Mérence. En effet, la version suivante passe aux aveux et tranche sur le doute référentiel, reconnaissant l'inexistence biographique de ce trait descriptif, et partant, son assignation tout arbitraire et toute fictive. C'est au prix de cette explicitation que peut réapparaître la détermination individualisante, sans risquer d'entamer sa valeur symbolique :

(A706) sur les genoux sacrés de mère en beauté jeune $<$ ne sont pas $>$

Qui n'étaient pas mais qui seront dans la genèse

Ce vers ajouté à la cinquième version (A706) fournit la raison des tergiversations de la visée référentielle, puisqu'aussi bien le poète semble reconnaitre à l'attribut de haut degré du « genou sacré » et de la « jambe immense » un statut plus symbolique que biographique. Sans doute en vertu de cette vision des proportions qui fait que, comme la chanson de Marie Paule Belle, «Dans les dessins d'enfant tout parait plus grand »...

Ce que peuvent les déterminants à l'endroit du nom commun, ils le peuvent également à l'endroit du nom propre. On a vu ce «Mère» pris entre une interprétation classificatoire ("des genoux de mère ») et une interprétation individualisante ("les genoux de mère »). Quant au vrai nom propre ("Antigone»), on sait qu'il n'a normalement pas besoin de l'article pour restreindre sa visée référentielle à l'individu singulier, car c'en est la vocation.

Or, dès le titre, cette valeur théoriquement individualisante, de «désignateur rigide » ${ }^{15}$, que devrait avoir le Nom propre est mise à mal par l'emploi d'un groupe déterminant pluralisant «les deux». Si l'Antigone mythique est une, alors qu'on compte beaucoup de Françoise et maintes Sophie, pluraliser Antigone consiste à dégager ce nom de son

\footnotetext{
${ }_{15}$ Dans sa théorisation du nom propre, Samuel Kripke soulignait la particularite du nom propre à s'employer «nu » (c'est-à-dire sans déterminant), puisqu'en vertu d'un «acte de baptême » consensuel et partagé, le nom avait pour vocation de ne désigner, de façon constante et unique, que l'individu auquel il avait été attribué ([Name and Necessity, 1972] La Logique des noms propres, trad. de l'anglais par Pierre Jacob et François Recanati, Paris, Minuit, 1982). Cet emploi purement désignationnel du nom propre fut longtemps le seul à intéresser les logiciens et continua d'influencer, à travers eux, les linguistes.
} 
ancrage référentiel singulier. Quelle valeur donner à cette démultiplication du nom?

Puisant dans la littérature relative à la détermination du nom propre, on pourrait théoriquement y attendre :

- une interprétation image ${ }^{16}$, si Antigone, seule et unique, laissait par exemple entrevoir différents visages personnels selon ses interlocuteurs. Il en est ainsi, par exemple, de la Jocaste bifrons qui, selon Bauchau, adresse un sourire irradiant vers un fils, cependant qu'elle tourne un visage sombre et nocturne vers son jumeau ;

- une interprétation métaphorique ${ }^{17}$ ferait prélever dans l'identité d'Antigone tel trait constitutif de son type, pour le reconnaittre dans tel autre personnage; Cécile est une vraie Antigone;

- si les femmes (et les hommes, comme Bauchau justement) capables des mêmes actes et des mêmes sacrifices qu'Antigone venaient à former une cohorte, et, partant, en termes linguistiques, une "classe d'entités ", la multiplication d'Antigone ferait du nom une véritable antonomase ${ }^{18}$.

De cette multiplication d'Antigone, je veux pour preuve pour le moins deux indices linguistiques tangibles : 1) la détermination hésitante des syntagmes, et donc une visée référentielle oscillant entre le classificatoire, voire le générique d'une part et d'autre part le spécifique ; et 2) la transformation qualificatoire d'« Antigone ».

1) Le flou désignationnel d'Antigone

Comme « Mère » qui, selon les constructions syntagmatiques, instruit une interprétation tantôt propriale, tantôt commune et générique, ainsi Antigone se dégage de sa propre chair mythique pour se faire trait distinctif, qualité notoire. Aussi peut-elle se réincarner, tour à tour dans telle île blanche aux parois lisses, tantôt encore, successivement ou

${ }^{16}$ Marie-Noëlle Gary-Prieur, Grammaire du nom propre, Paris, Larousse, 1994.

17 Georges Kleiber, Nominales. Essais de sémantique référentielle, Paris, Armand Colin, "Linguistique », 1994 ; Kerstin Jonasson, Le Nom propre. Constructions et interprétations, Louvain-La-Neuve, Duculot, «Champs linguistiques », 1994.

18 Sarah Leroy, De l'identification à la catégorisation. L'antonomase du nom propre en français, Louvain/Paris, Dudley MA/Peeters, 2005. Pour une synthèse des emplois figurés du nom propre et la bibliographie relative, voir Geneviève Henrot Sostero, Pragmatique de l'anthroponyme dans À la recherche du temps perdu de Marcel Proust, Paris, Champion, « Lexica » n²0, 2011. 
simultanément, dans tel ou tel autre personnage ${ }^{19}$. Cette potentielle démultiplication par réincarnations successives explique le -s morphème de pluriel qui apparaît spontanément sur une des versions manuscrites du titre: "Les deux Antigones» (A 710). Mais le mouvement de désincarnation va plus loin encore : il pousse le nom à quitter l'individu pour le type (on vient de le voir), mais aussi le type pour la simple qualité.

2) Valeur qualificative d'Antigone (Mère, Mérence, moi) ${ }^{20}$

Pour comprendre ce pouvoir d'assimilation ou d'amalgame qu'a Antigone, il est utile d'observer son comportement syntaxique récurrent : le mot apparait à de nombreuses reprises, dans le poème et dans le reste de l'œuvre, en fonction prédicative tantôt d'épithète, tantôt d'attribut. Cette construction syntaxique fonctionnelle de l'unité linguistique a le pouvoir de lui faire changer de classe, et, en lui ôtant son rôle de référenciation au profit de la prédication, d'en faire une valeur purement sémantique, de qualification, une espèce d'adjectif par conversion. Des syntagmes tels que :

La statue Antigone

La lumière Antigone ${ }^{21}$

L'ombre Antigone

pourraient se paraphraser comme suit : « une statue qui a les qualités propres à Antigone », "une lumière qui a des qualités semblables au caractère d'Antigone » etc. Il est du reste connu, en linguistique, que le

${ }^{19}$ Comme dans le roman homonyme, quand Antigone se confond avec Io, elle devient «l'Antigone d'Io » (A, p. 354).

${ }^{20}$ Nadège Coutas, «Le paradoxe Antigone dans le roman d'Henry Bauchau. Figuration de l'exil », Revue internationale Henry Bauchau, n³, 2010, pp. 138-149.

${ }^{21}$ Il a été remarqué combien l'opposition du clair et de l'obscur, de la lumière et de l'obscurité sous-tend le roman d'un bout à l'autre: Yves Leroux, «Ombres et lumière dans l'Antigone d'Henry Bauchau: approche cinématographique », dans Revue internationale Henry Bauchau, n², 2009, pp. 112-115; «À ce moment on découvre que la vie $[\ldots]$ demeure le vrai trésor et que sa lumière, voilée par les nuages du temps et de l'épreuve, nous éclaire toujours » ( $J A$, p. 501). Le Journal d'Antigone confirme largement qu'ombres et lumières n'ont cessé d'assaillir l'auteur tout au long de la gestation du roman. 
nom propre peut occuper la place et remplir la fonction d'épithète, selon la formule neutralisée [Dét. $\left.+\mathrm{N}_{1}+\mathrm{N}_{2}\right]^{22}$.

Lorsqu'il y a identité coréférentielle entre $\mathrm{N}_{1}$ et $\mathrm{N}_{2}$, l'épithète pourrait s'interpréter comme une apposition $\left(\mathrm{N}_{1}\right.$ Statue $=\mathrm{N}_{2}$ Antigone, $\mathrm{N}_{1}$ lumière $=\mathrm{N}_{2}$ Antigone), à en croire, par exemple, le Journal d'Antigone: "Antigone n'est pas une espérance, c'est une lumière » (JA, 275). Partant, on observe ce qui unit l'épithète et l'apposition à une autre fonction, celle de l'attribut: c'est la prédication, la faculté de prédiquer quelque chose à propos d'un thème. La position droite $\mathrm{N}_{2}$ (épithète, apposition ou attribut) apporte au thème posé par le substantif $\mathrm{N}_{1}$ une prédication de qualité qui permet de l'identifier singulièrement dans le vaste monde référentiel. Elle permet d'établir des équivalences et des identités, comme dans « La statue Antigone».

C'est donc en faisant d'Antigone une qualité d'etre que Bauchau parvient, en accord avec les potentialités de la langue, à produire et à dire non seulement la fusion des trois femmes, mais aussi l'amalgame des genres, ainsi : "Je suis devenu Antigone ${ }^{23}$. L'identification seulement spirituelle et affective s'est déprise des fallacieuses enveloppes corporelles responsables d'une définition sexuelle. Les équivalences paradigmatiques, les équations fonctionnelles, la fluctuation de la visée référentielle et la conversion prédicative du Nom propre permettent à «Antigone» de se multiplier et de rallier à son mythe tout le personnel du poème par la voie de l'intériorité, par ce que Bauchau, relisant son poème quelques années plus tard, a appelé «la présence de l'inconnu intérieur ».

Par le biais d'une analyse syntaxique, sémantico-référentielle et actancielle centrée sur la reprise de séquences syntagmatiques inférieures ou égales à l'unité phrastique, l'attention portée sur un menu avant-texte de poème a permis de montrer d'abord combien les personnes du poème

\footnotetext{
22 Que $\mathrm{N}_{1}$ ou $\mathrm{N}_{2}$ équivalent eux-mêmes à un nom propre ou à un nom commun, Michèle Noailly, Le substantif épithète, Paris, PUF, 1990.

${ }^{23}$ Subtilement le psychanalyste précise que, tout en la sentant très proche, il n'a « jamais cessé de percevoir en Antigone le mystère de la femme pour l'homme» (dans Yves Leroux, art. cit., p. 113). Et Bauchau d'insister: «Pour suivre Antigone, pour la comprendre, j'ai fait appel à la part féminine qui existe en moi [...] J'ai fait appel aussi, dans Antigone [...] à la part virile existant en elle comme en toute femme [...] j'ai poussé Antigone [...] vers une certaine image androgyne » $(J A$, p. 500).
} 
présentent des identifications ambiguës ou interchangeables, créant un faisceau de traits communs qui invite à superposer des textes aussi distants que «L'Escalier bleu» et «Les deux Antigone» comme des versions ou des reformulations l'un de l'autre, ou des variations sur le thème. Si cette polysémie du personnage ne fait pas nouveauté, du moins est-il intéressant de voir comment elle «bouge» dans les brouillons, au fil des réécritures, des phrases inachevées et inlassablement reprises, sur un même feuillet ou d'un feuillet l'autre. Elle a montré ensuite combien Antigone est un rôle profond (adjuvant), un prédicat qualificatif civique, indépendant de toute incarnation civile, susceptible de se glisser dans la peau du je (c'est lui la deuxième Antigone). D'où le sens de l'incipit: l'un pour l'autre, l'un et l'autre, l'un dans l'autre.

"Antigone est une des rencontres de ma vie» disait Henry Bauchau (JA, p. 509). «L'ombre dont nous sommes faits est peut-être exquise. Mais chacun, dit Clios à Antigone, doit faire naître son soleil ». C'est ce que réalise le poème Les deux Antigone.

Je relis ce matin Les deux Antigone, et je suis frappé par les vers où Antigone dit à CEdipe qu'elle l'a guidé « vers le dieu qui mendie ». Il y a donc eu une rencontre de deux mendiants, l'un présent et l'autre invisible. Étrange que je le comprenne seulement aujourd'hui, alors que c'est en 1982 que j’ai écrit ce poème qui m'indiquait la voie. En découvrant cela, j’ai ressenti une émotion submergeante que je connais bien. Émotion qui est la source, en moi, de l'écriture et du signe de la présence de l'inconnu intérieur. (JJ, p. 132.)

Geneviève HENROT

Université de Padoue 